RESEARCH PAPER

\title{
Impact of UK National Health Service smoking cessation services: variations in outcomes in England
}

\author{
L Bauld, J Chesterman, K Judge, E Pound, T Coleman, on behalf of the English \\ Evaluation of Smoking Cessation Services (EESCS)
}

Tobacco Control 2003;12:296-301

\begin{abstract}
Objectives: To determine the extent to which UK National Health Service (NHS) smoking cessation services in England reach smokers and support them to quit at four weeks, and to identify which service and area characteristics contribute to observed outcomes.

Design: Ordinary least squares regression was used to investigate local smoking outcomes in relation to characteristics of health authorities and their smoking cessation services.

Setting: 76 health authorities (from a total of 99) in England from April 2000 to March 2001.

Main outcome measures: Reach-number of smokers attending cessation services and setting a quit date as a percentage of the adult smoking population in each health authority. Absolute success-number of smokers setting a quit date who subsequently reported quitting at four weeks (not having smoked between two and four weeks after quit date). Cessation rate-number of smokers who reported quitting at four weeks as a percentage of those setting a quit date. Loss-percentage lost to follow up.

Results: A range of service and area characteristics was associated with each outcome. For example, group support proved more effective than one to one interventions in helping a greater proportion of smokers to quit at four weeks. Services based in health action zones were reaching larger numbers of smokers. However, services operating in deprived communities achieved lower cessation rates than those in more prosperous areas.

Conclusions: Well developed, evidence based NHS smoking cessation services, reflecting good practice, are yielding positive outcomes in England. However, most of the data are based on self reported smoking status at four weeks. It will be important to obtain validated data about continuous cessation over one year or more in order to assess longer term impact.
\end{abstract}

See end of article for authors' affiliations

Correspondence to: Professor Ken Judge, Health Promotion Policy

Unit, University of

Glasgow, 1 Lilybank

Gardens, Glasgow

G12 8RZ, UK;

K.Judge@clinmed.gla.ac.uk

Received

4 October 2002

Accepted 21 April 2003

t

n 1998, the UK government outlined new policies to combat tobacco addiction in the White Paper Smoking kills. ${ }^{1}$ A key component of the strategy included the creation of the first national network of smoking cessation services as part of the National Health Service (NHS). The establishment of NHS smoking cessation services represents a unique national experiment to assist smokers to stop, and as a result has attracted the interest of observers from other countries. The experience of services in the UK, and the extent to which they are successful in helping smokers to quit, could yield valuable lessons that are applicable in an international context.

In England, funding was made available to health authorities to establish services from 1999 in some of the most disadvantaged parts of the country with high smoking prevalence rates (health action zones (HAZs)). From April 2000 services were funded across the country. The progress made by services is being monitored. The Department of Health (DoH) expects cessation services to collect basic information from each smoker who sets a quit date. Further data are collected at four weeks to determine whether the smoker has quit. In addition to basic monitoring, a national evaluation was commissioned by the DoH from November 2000, to which this paper contributes.

Our paper aims to describe how variations in health authority and service characteristics account for observed differences in outcome. In particular, analyses examined the extent to which factors such as area deprivation and smoking prevalence are relevant, and investigated whether particular elements of service structure, such as location, leadership, and type of intervention affected outcomes.

\section{METHODS}

Smoking cessation services are required to submit aggregate quarterly monitoring data to the DoH. For the purpose of this analysis the monitoring returns for 2000-2001 were linked with data about:

(1) area deprivation in health authorities ${ }^{2}$

(2) smoking prevalence rates for health authorities between 1994 and $1996^{3}$

(3) adult population ( 16 years and over) for health authorities in $1996^{4}$

(4) data from a postal questionnaire of smoking cessation service coordinators for health authorities conducted between March and May 2001.5

\section{Coordinator survey data}

A list of smoking cessation coordinators for all health authorities was obtained from the regional offices of the DoH. Out of 133 questionnaires dispatched, six were returned because the named coordinator was not known or had moved. Of the remaining 127 questionnaires, 69.3\% (88) were returned. At least one completed questionnaire was received from $83 \%$ of England's health authorities. This survey ${ }^{5}$ produced a database with the coordinator as the unit of analysis. The database was converted in the present study into one for which the health

Abbreviations: DoH, Department of Health; $\mathrm{HAZ}$, health action zone; $I M D$, index of multiple deprivation; NHS, National Health Service; OLS, ordinary least squares; PCGs/PCTs, primary care groups/trusts 
Table 1 Distributions of dependent variables used in ordinary least squares (OLS) regression modelling

\begin{tabular}{|c|c|c|c|c|c|c|c|c|}
\hline \multirow[b]{2}{*}{ Outcomes } & \multicolumn{4}{|c|}{$\mathrm{n}=99$ health authorities* } & \multicolumn{4}{|c|}{$\mathrm{n}=76$ health authorities } \\
\hline & Min & Max & Mean & $\begin{array}{l}\text { Coefficient } \\
\text { of variation } \ddagger\end{array}$ & Min & $\operatorname{Max}$ & Mean & $\begin{array}{l}\text { Coefficient } \\
\text { of variation } \ddagger\end{array}$ \\
\hline $\begin{array}{l}\text { Reach: } n \text { users setting quit date as } \% \text { of smoking adult } \\
\text { population } 16+\end{array}$ & 0.03 & 5.51 & 1.26 & 91.1 & 0.03 & 5.51 & 1.39 & 85.3 \\
\hline Absolute success: $\mathrm{n}$ users successfully quitting at 4 weeks & 9 & 2147 & 652 & 83.2 & 9 & 1992 & 697 & 75.7 \\
\hline $\begin{array}{l}\text { Cessation rate: Cessation rate at } 4 \text { weeks as } \% \text { of those setting } \\
\text { a quit date }\end{array}$ & 22.3 & 78.6 & 52.5 & 22.9 & 26.9 & 74.1 & 51.6 & 19.9 \\
\hline Loss: Loss rate at 4 weeks as $\%$ of those setting a quit date & 0.0 & 71.5 & 21.1 & 64.9 & 3.2 & 60.2 & 21.1 & 55.1 \\
\hline
\end{tabular}

authority was the unit of analysis. There was normally no difference, the coordinator covering the whole health authority. When several coordinators operated from different bases, values for particular variables were aggregated where possible or set to missing when in disagreement, producing a coordinator database relating to 76 health authorities, out of the total of 99 in England. In the four health authorities with more than one coordinator, one quarter of the data were missing because of disagreement between coordinators.

\section{Measures}

From the monitoring data for 2000-1, four smoking cessation outcomes were derived:

(1) percentage of smoking adult population accessing the service and setting a quit date (reach)

(2) number who reported quitting at four weeks-defined by the DoH as not having smoked between two and four weeks after quit date (absolute success)

(3) number who reported quitting at four weeks as a percentage of those setting a quit date (cessation rate)

(4) percentage of service users lost to follow up at four weeks (loss).

Considerable efforts were made by local services to collect carbon monoxide (CO) validated information about cessation, but this proved to be very difficult. Data about four week quit rates, therefore, were largely self reported.

The four smoking cessation outcomes are explored in relation to a range of measures that might be associated with them. Core characteristics of the health authority were adult population, its distribution by age band and sex, smoking prevalence rate, whether an HAZ, and index of multiple deprivation (IMD). The IMD is an overall measure of deprivation for every electoral ward and local authority area in England. It combines a number of indicators, that cover a range of domains (income, employment, health deprivation and disability, education skills and training, housing and geographical access to services), into a single deprivation score for each area. ${ }^{2}$ Measures derived from the smoking cessation coordinator database related to employees of the service, the structure and location of the service, and the interventions delivered.

\section{Data analysis}

First, we illustrate the overall extent of variation between health authorities in relation to the four outcome measures of interest. We also show the range of values for the variables used to predict outcomes. The relation between outcomes and predictor variables was investigated using ordinary least squares (OLS) regression. Such analyses were only possible for approximately three quarters of all health authorities $(n=76)$ where reasonably full information about services was available from survey data. ${ }^{5}$ The data for the outcome variables related to reach, absolute success, and loss have been log transformed (base 10) so as to approximate normal distributions; cessation rate was already normally distributed. Standardised residuals then showed no strong dependency on predicted value. Significant characteristics of health authorities and smoking cessation services at the 5\% level were identified using forward stepwise linear regression analysis. Finally, we estimated the relative importance of area and service characteristics as determinants of smoking cessation outcomes. Further details about methods and sensitivity analyses are contained in a technical report $t^{6}$ and correlations between variables are shown in an appendix, which is available in the electronic version of Tobacco Control.

\section{RESULTS}

Considerable progress was made in providing smoking cessation services in England during 2000-1. More than 132500 smokers attending cessation services set a quit date and 53\% of these were reported as having stopped smoking on follow up at four weeks. ${ }^{7}$ At the local level, however, there was considerable variation between health authorities. Table 1 compares data from all health authorities $(n=99)$ with the sample $(n=76)$ for which more detailed data from the coordinator survey were available. It shows the degree of variation in relation to the four key outcomes of interest.

The findings for the sample are almost identical to those for English health authorities as a whole. Most variation was found in relation to the number of users setting a quit date. There was also considerable diversity in number of successes and in the proportion of smokers setting a quit date who were lost to follow up at four weeks. Considerably less variability was observed in relation to cessation rates.

Table 2 shows the degree of variation among the set of predictor variables entering one or more of the multivariate statistical models for the reduced sample of 76 health authorities.

Table 3 reports the results of multivariate OLS models, which show that a small number of area characteristics and a wider range of service characteristics were significantly associated with each outcome measure. For each term in the model, the unstandardised $\beta$ coefficient is shown, with its significance level in brackets.

\section{Reach}

Services in HAZs, which were located in areas of high deprivation, were reaching on average some $140 \%$ more smokers than those in other parts of the country. Smoking prevalence rate did not enter the model.

Several service characteristics also influenced the capacity to reach larger numbers of smokers. Not surprisingly, as the number of months before the coordinator anticipated the 
Table 2 Distribution of predictor variables entering ordinary least squares regression models*

\begin{tabular}{|c|c|c|c|c|}
\hline Predictor variable & Minimum & Maximum & Mean & $\begin{array}{l}\text { Coefficient } \\
\text { of variation } \dagger\end{array}$ \\
\hline \multicolumn{5}{|l|}{ Area characteristics } \\
\hline Whether an $\mathrm{HAZ}$ & 0 & 1 & 0.39 & 124.7 \\
\hline Index of multiple deprivation & 7.9 & 58.0 & 27.8 & 40.0 \\
\hline Number adult smokers $16+$ in health authority (millions) & 0.033 & 0.226 & 0.115 & 37.3 \\
\hline$\%$ population aged $30-44$ & 23.9 & 35.3 & 27.8 & 7.8 \\
\hline \multicolumn{5}{|l|}{ Service characteristics } \\
\hline Number of months before service expected to work at full capacity & 0 & 15 & 2.26 & 147.0 \\
\hline Service operating at full capacity now & 0 & 1 & 0.61 & 79.5 \\
\hline Coordinator has responsibilities other than developing/running service & 0 & 1 & 0.56 & 87.0 \\
\hline Salary level of coordinator & 1 & 2 & 1.53 & 32.4 \\
\hline Difficulty recruiting smoking cessation service advisors or counsellors & 1 & 5 & 2.78 & 36.3 \\
\hline Difficulty finding good quality training courses for advisors or counsellors & 1 & 5 & 2.46 & 47.6 \\
\hline Central base used (almost) exclusively by smoking cessation service & 0 & 1 & 0.11 & 280.9 \\
\hline Satellite/outreach bases shared between service and other organisations & 0 & 1 & 0.81 & 47.9 \\
\hline General practices sometimes used by advisors to deliver cessation interventions & 0 & 1 & 0.89 & 34.7 \\
\hline Other NHS primary care premises used (other than general practices, pharmacies) & 0 & 1 & 0.68 & 68.5 \\
\hline Percentage of users receiving individual support & 0 & 100 & 46.5 & 67.2 \\
\hline Length (minutes) of individual sessions & 12 & 60 & 27.9 & 36.2 \\
\hline Number of individual sessions in a complete treatment course & 2 & 8 & 5.3 & 29.0 \\
\hline$\%$ users receiving group support & 0 & 100 & 55.0 & 56.5 \\
\hline Number of different types of professionals receiving training from service staff & 0 & 7 & 5.4 & 30.1 \\
\hline Protocol for dispensing or prescribing bupropion agreed with local GPs & 0 & 1 & 0.82 & 46.1 \\
\hline Whether advisors/counsellors ever recommended bupropion & 0 & 1 & 0.81 & 46.4 \\
\hline Strength of relationship of services with local PCGs/PCTs & -4 & -1 & -2.19 & 39.2 \\
\hline
\end{tabular}

service to start "operating at full capacity" increased, the reach diminished. When this amounted to 12 or more months the reduction in reach was $70 \%$. The percentage of users receiving individual (one to one) smoking cessation interventions was positively associated with reach. Thus a $10 \%$ increase in the percentage of users receiving individual support was accompanied by a $6 \%$ increase in reach. Finally, the stronger the relationship of services with local primary care organisations (primary care groups/trusts (PCGs/PCTs)), the greater the reach.

\section{Absolute success}

The number of people who reported quitting at four weeks was $90 \%$ greater in HAZ areas and was also larger when the service was operating at full capacity. Other factors that contributed to a larger numbers of quitters included longer individual sessions and stronger service relationships with local PCGs/PCTs. An increase of 10 minutes in the length of an individual session was accompanied by a $20 \%$ increase in the number of successes. When the relationship with local PCGs/ PCTs was described as "strong", the number of successes was some $40 \%$ greater than when classed as "weak". In contrast, the number of successful quitters was lower when the service had experienced staffing problems such as difficulty recruiting smoking cessation service advisors or counsellors.

\section{Cessation rate}

Services operating in deprived parts of the country achieved lower cessation rates than those in more affluent areas. Typically, in moving from an area with a deprivation score at the lower quartile (19) to one at the upper quartile (36), the reduction in cessation rate would be $6 \%$. In terms of service characteristics, lower levels of cessation were found if the smoking cessation coordinator had responsibilities other than running the service. Some coordinators occupied this position on a part time basis while combining it with other clinical or managerial responsibilities. This was accompanied by a $6 \%$ reduction in the cessation rate. Location also emerged as important in terms of reported quitting: services were less able to support smokers to quit at four weeks if they were operating primarily from one central location in contrast to a range of locations, this amounting to a reduction of $10 \%$ in the cessation rate. The use of general medical practices as a venue for trained advisers to deliver smoking cessation interventions, however, was positively associated with quitting.

Three aspects of treatment were positively associated with cessation at four weeks. The proportion of service recipients receiving group (rather than one to one) support was very significantly associated with success. Thus, a $10 \%$ increase in this proportion was accompanied by an increase of $2 \%$ in the cessation rate. When one to one interventions were received, however, quit rates were enhanced by the number of individual sessions in a complete treatment course. If a protocol for dispensing or prescribing bupropion was agreed with local general practitioners, this also increased cessation rates.

\section{Loss rate}

Services operating in deprived areas were more likely to lose contact with smokers between the period of setting a quit date and reporting outcomes at four weeks. In addition, a wide range of service characteristics was associated with loss to follow up. Issues of leadership emerged as significant: services whose coordinator had responsibilities other than running the service were more likely to lose smokers, with loss rates $7 \%$ greater; in contrast, services whose coordinator was paid a higher salary were less likely to lose smokers. Staff issues were again a problem. Difficulty finding good quality training courses for advisors or counsellors was associated with higher loss rates. Again location was important: services operating exclusively from one central base were more likely to incur higher loss rates. In contrast, those offering a range of satellite or outreach locations were better able to retain smokers.

A range of treatment characteristics was significant in relation to loss to follow up. The percentage of users receiving individual support was positively associated with loss to follow up: thus a $10 \%$ increase in the proportion of users receiving individual support was accompanied by an increase of $2 \%$ in loss rate. However, longer individual sessions appeared to protect against some users leaving the service. Services that trained a range of professionals as smoking cessation advisers were less likely to lose smokers. Finally, services whose advisers 
Table 3 Determinants of smoking cessation outcomes

\begin{tabular}{|c|c|c|c|c|}
\hline \multirow[b]{2}{*}{ Predictor variables (ordinary least squares estimation) } & \multicolumn{4}{|c|}{ Smoking outcomes: unstandardised coefficient (significance level) } \\
\hline & Reach (\%) & $\begin{array}{l}\text { Absolute success } \\
\text { (n) }\end{array}$ & $\begin{array}{l}\text { Cessation } \\
\text { rate }(\%)\end{array}$ & Loss (\%) \\
\hline \multicolumn{5}{|l|}{ Area characteristics } \\
\hline Whether an HAZ & $0.593(0.001)$ & $0.51(0.001)$ & & \\
\hline Index of multiple deprivation & & & $-0.35(0.001)$ & $0.0051(0.006)$ \\
\hline Number of adult smokers aged $16+$ in health authority (millions) & & $2.3(0.001)$ & & \\
\hline$\%$ population aged $30-44$ & & $-0.027(0.024)$ & & \\
\hline \multicolumn{5}{|l|}{ Service characteristics } \\
\hline Number of months before service expected to work at full capacity & $-0.024(0.009)$ & & & \\
\hline Service operating at full capacity now & & $0.13(0.015)$ & & \\
\hline Coordinator has responsibilities other than developing/running service & & & $-6.3(0.001)$ & $0.15(0.001)$ \\
\hline Salary level of coordinator & & & & $-0.088(0.037)$ \\
\hline Difficulty recruiting smoking cessation service advisors or counsellors & & $-0.053(0.043)$ & & \\
\hline Difficulty finding good quality training courses for advisors or counsellors & & & & $0.037(0.042)$ \\
\hline Central base used (almost) exclusively by smoking cessation service & & & $-10.3(0.001)$ & $0.14(0.043)$ \\
\hline Satellite/outreach bases shared between service and other organisations & & & & $-0.12(0.029)$ \\
\hline $\begin{array}{l}\text { General practices sometimes used by advisors to deliver cessation } \\
\text { interventions }\end{array}$ & & & $7.5(0.013)$ & \\
\hline $\begin{array}{l}\text { Other NHS primary care premises used (other than general practices, } \\
\text { pharmacies) }\end{array}$ & & $0.130(.047)$ & & \\
\hline$\%$ users receiving individual support & $0.0028(0.004)$ & & & $0.0035(0.001)$ \\
\hline Length (minutes) of individual sessions & & $0.0076(0.004)$ & & $-0.0066(0.002)$ \\
\hline Number of individual sessions in a complete treatment course & & & $1.3(0.018)$ & \\
\hline$\%$ users receiving group support & & & $0.18(0.001)$ & \\
\hline $\begin{array}{l}\text { Number of different types of professionals receiving training from service } \\
\text { staff }\end{array}$ & & & & $-0.035(0.008)$ \\
\hline Protocol for dispensing or prescribing bupropion agreed with local GPs & & & $5.5(0.016)$ & \\
\hline Whether advisors/counsellors ever recommended bupropion & & & & $-0.15(0.012)$ \\
\hline Strength of relationship of services with local PCGs/PCTs & $0.073(0.041)$ & $0.081(0.008)$ & & \\
\hline Constant & $-0.17(0.091)$ & $2.96(0.001)$ & $38.2(0.001)$ & $1.52(0.001)$ \\
\hline Sample size & 74 & 75 & 74 & 74 \\
\hline$R^{2}$ & 0.65 & 0.71 & 0.54 & 0.53 \\
\hline Adjusted $\mathrm{R}^{2}$ & 0.63 & 0.67 & 0.49 & 0.46 \\
\hline Significance level of $F$ & $<0.001$ & $<0.001$ & $<0.001$ & $<0.001$ \\
\hline
\end{tabular}

recommended bupropion in appropriate circumstances demonstrated lower rates of loss to follow up.

\section{Relative importance of predictors}

In order to determine the proportion of the variance in the four models that was explained by area and service characteristics, each model was rerun with area characteristics alone. These accounted for a large proportion of the variation in relation to reach $(81 \%)$ and the absolute number of successful self reported four week quits (79\%). This largely reflects the more highly developed and better funded services available in HAZs, allowing more smokers to be reached. However, service characteristics were substantially more important in accounting for the cessation rate (78\%) and loss to follow up (98\%).

\section{DISCUSSION}

When this study was conducted, NHS smoking cessation services were relatively new. Early results suggest that services were successful in reaching smokers and supporting them to quit, but illustrate that the factors influencing outcomes are many and varied. In particular, there is considerable variation between the four outcomes examined in this study: reach, absolute success, cessation rate, and loss to follow up. Most variation was found in relation to the number of users setting a quit date, which reflected the very variable rate of development of services during 2000-1, and also the fact that services in HAZs received a higher level of funding than those in other areas. The strong inverse relation between reach and cessation rate suggests that when more effort went into attracting a large number of smokers to the service, this tended to be at the expense of cessation rates. However, the overall pattern of results suggests that high reach is desirable if the primary objective is to maximise the actual number of people who stop smoking.

Many of the factors that emerged as significant determinants of reach, absolute success, cessation rate, and reduced loss to follow up could be described as characteristics of "good practice". These fell into three main categories: stage of development; leadership; and form of intervention.

Cessation services that were still appointing staff, organising training and establishing referral pathways during the course of 2000-1 were less likely to be treating large numbers of users. Thus the finding that services that were working at full capacity were better able to reach smokers is not surprising. Services in HAZ areas had been in place since 1999 and thus were at a later stage of development that those in other health authorities. These services also received a higher level of funding. This may partially explain their ability to reach larger numbers of smokers. In addition, services that encountered implementation problems, such as difficulties appointing staff, achieved fewer four week quits. Other findings, we believe, are indicative of the stage of service development. For instance, services that had trained a range of professionals in smoking cessation demonstrated lower rates of loss to follow up. This could indicate either greater success in supporting smokers or greater administrative efficiency in information retrieval. The ability to offer training to pharmacists, practice nurses, health visitors, midwives, and others was characteristic of a service that was aiming to reach and support smokers in a range of settings, in particular the target groups specified in Smoking kills. ${ }^{1}$

In order for services to develop further, issues such as leadership are also important. Findings suggested that a dedicated smoking cessation coordinator, earning an appropriate salary, yielded positive outcomes; in particular that the cessation rate 
increased and fewer smokers were lost to follow up. This reduced loss rate may reflect greater dexterity in monitoring smokers as well as an improved cessation rate. It was hardly surprising that setting up and managing a new service should be a full time job. A coordinator, who was able to act as an advocate for the service, as well as manage and motivate staff, could be invaluable. The ability to forge strong links with local PCGs/PCTs was also important.

The type of intervention offered by services also emerged as a determinant of positive outcomes. Here the link with established evidence regarding effective methods of cessation support was clear. Services that had established a system of working with local general practitioners to prescribe bupropion to smokers when appropriate, and combined it with support from a trained adviser (through the use of a protocol), demonstrated higher reported quit rates at four weeks. Similarly, services whose advisers could recommend bupropion to smokers (again when appropriate) appeared to have lower rates of loss to follow up. These results are consistent with recent findings from the UK National Institute for Clinical Excellence's review of nicotine replacement therapy and bupropion as smoking cessation aids, ${ }^{8}$ which found that both were highly effective. Data here related to the period before nicotine replacement therapy became available on prescription in the UK (from April 2001), which may partially explain why service factors associated with its use did not emerge as significant predictors of cessation. Instead, our findings suggest that bupropion, when prescribed appropriately and combined with motivational support from a trained adviser, may help smokers to quit. ${ }^{89}$

The proportion of users receiving group support was a predictor of four week quit rates. Group interventions were likely to be offered by specialist advisers who had the skills and knowledge to motivate smokers to sustain their quit attempt. One to one interventions were linked to number of successes and reduced loss rates only when sessions involved sustained contact between adviser and smoker, rather than shorter interventions. It is interesting to note that, in contrast, the percentage of users receiving one to one sessions was associated with both more effective reach and higher rates of loss to follow up. It may be that most smokers initially contacting a service preferred the convenience of one to one meetings with an adviser in a nearby location, but that these interventions were less effective than group support in sustaining attendance and delivering quit rates. However, the higher loss rate may simply reflect inefficiency in monitoring users rather than less effective support.

The reason that smoking prevalence rate did not enter the model for reach was due to confounding. Prevalence rates were highest in the most disadvantaged areas. The inclusion of HAZ status, therefore, probably captured most of the variation in smoking rates between health authorities. Deprivation was negatively associated with cessation at four weeks. The more limited cessation rates in disadvantaged areas may in fact be due to the particular challenges inherent in supporting low income smokers to quit. As other research has indicated, quitting can be extremely difficult for smokers living in deprived areas. ${ }^{10}$ In many cases these smokers may be more dependent upon nicotine and require more intensive interventions.

It is important to emphasise that four week reported quit rates do not automatically translate to continuous abstinence. If the factors contributing to success rates are to be more comprehensively evaluated, data about longer term outcomes are required. As things stand, this may be extremely difficult to obtain for NHS smoking cessation services. While services were initially required to collect 52 week quit rates for specialist services as part of the DoH's monitoring requirements, this is no longer mandatory. Monitoring results published by the DoH suggested that 52 week quit rates were low because of high rates of loss to follow up and

\section{What this paper adds}

There is considerable evidence from clinical trials and national guidelines that smoking cessation interventions are effective-for example, brief advice supported by specialist smoking cessation support.

There is no existing evidence regarding which factors will influence the impact or effectiveness of services when rolled out on a national basis. This paper aims to shed some light on this issue, in the context of the UK.

other factors-13\% from specialist services in HAZs in 2000$1 .^{7}$ At least part of this was because of the real practical difficulties that services were encountering in contacting smokers one year on. The implication is that routine clinical services are inadequate for collecting such data, and that a more labour intensive approach with dedicated staff is required in order to minimise loss rates. Still more problematic is the lack of comprehensive $\mathrm{CO}$ verification at follow up. Experience shows that self reported quit rates tend to be overestimated by a factor that probably varies with locality. Because self reported quitting is not always accurate, further studies should, where possible, obtain CO validation.

\section{Conclusions}

During 2000-1, the new smoking cessation services achieved substantial success in helping smokers to quit at four week follow up. However, the level of success was not uniform across the country. In HAZs, better outcomes were observed. HAZs were provided with funding one year earlier than services in other parts of the country and thus higher quit rates in these areas were due at least in part to their well established, well funded services. A number of other findings suggest that stage of service development was a significant factor in influencing both reach and cessation rates. This suggests that this study should be replicated now that services are fully established across England.

A number of specific service characteristics emerged as significant predictors of reach, absolute success, and cessation rates. In particular, services that had a dedicated, full time coordinator achieved better outcomes. One to one support to smokers improved reach, but services offering group sessions demonstrated higher quit rates. Cessation rates for one to one sessions were greater when more treatment sessions were offered. In addition, services that had good relationships with local primary care organisations demonstrated both higher reach and a greater number of successes.

This study is limited because of its reliance on self reported four week quit data. This reflects the practical reality of evaluating smoking cessation in everyday service settings, rather than in trials. The current absence of data regarding longer term, CO validated quit rates is a cause for concern. The success of English smoking cessation services cannot be judged on self reported four week quit rates alone. If valuable lessons are to be learnt from this investment in a national network of services, greater efforts need to be made at both local and national level to measure longer term cessation, ideally using CO validation. Only then will it be possible to evaluate the contribution that cessation services have made to the pursuit of the ambitious targets for reducing smoking prevalence set out in Smoking kills.

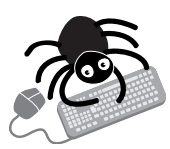

To see the appendix, go to the Tobacco Control website-www.tobaccocontrol.com/supplemental 


\section{ACKNOWLEDGEMENTS}

This research was funded by the Department of Health. The views expressed in this article are those of the authors and not necessarily those of the Department of Health. The authors wish to thank Dr Ann McNeill, Dr Martin Raw, Professor Robert West, and two anonymous referees who provided comments on earlier versions of this article

\section{Authors' affiliations}

L Bauld, Department of Social Policy and Social Work, University of Glasgow, Glasgow, UK

J Chesterman, K Judge, Health Promotion Policy Unit, University of Glasgow

E Pound, Department of General Practice and Primary Health Care, University of Leicester, Leicester, UK

T Coleman, Department of General Practice, University of Nottingham, Nottingham, UK

\section{REFERENCES}

1 Department of Health. Smoking kills: a white paper on tobacco. London: Department of Health, 1997. URL: http://www.doh.gov.uk.

2 Department of the Environment, Transport and the Regions. Indices of deprivation 2000. Regeneration Research Summary Number 31. London: Department of the Environment, Transport and the Regions, 2000. URL: http://www.dtlr.gov.uk
3 Department of Health. Health survey for England: geographical variation in health indicators by health authority, 1994-1996, introductory guide and data definitions. London: Department of Health 1999. URL: http://www.doh.gov.uk

4 Office for National Statistics. Dataset on population and migration: 1996-based English subnational population projections: Health Authorities 1996. London: Department of Health, 1996. URL: http://www.doh.gov.uk

5 Pound E, Coleman T, Cheater F, et al, on behalf of the English Evaluation of Smoking Cessation Services Team. National survey of the new smoking services in England. Health Education Journal (in press).

6 Chesterman J, Judge K, Bauld L, et al. Effectiveness of smoking cessation services: an analysis of outcomes at the health authority level. Report submitted to the Department of Health, July 2002. URL: http://www.dph.gla.ac.uk/hppu/publications.htm

7 Department of Health. Statistics on smoking cessation services in England: April 2000-March 2001. London: Government Statistical Service, 2001.

8 National Institute for Clinical Excellence. Guidance on the use of nicotine replacement therapy (NRT) and bupropion for smoking cessation. Technology Appraisal Guidance No. 39. London: NICE, 2002.

9 Raw M, McNeill A, West R. Smoking cessation guidelines and their cost-effectiveness. Thorax 1998;53(suppl 5).

10 Jarvis $M$, Wardle M. Social patterning of health behaviours: the case of cigarette smoking. In Marmot M, Wilkinson R, eds. Social determinants of health. Oxford: Oxford University Press, 1999.

\section{The Lighter Side}

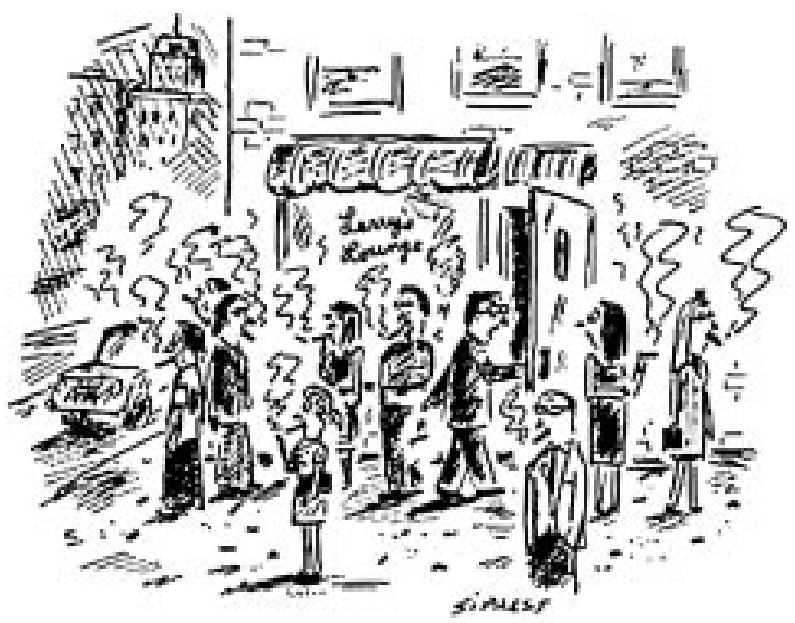

-I'm going back inside to get some air."

(OThe New Yorker Collection. David Sipress from Cartoonbank.com. All rights reserved. 